\title{
Alta utilidad de monitorización hemodinámica con ecocardiografía transesofágica intraoperatoria en cirugía ortopédica
}

\author{
María Carolina Cabrera Sch ${ }^{1,2}$, Raúl Vega $S^{1}$, \\ Victoria Díaz Valdés $A^{1}$, Ernesto Bermúdez $B^{1}$, \\ Emilio Santelices $C^{1}$. \\ Intraoperative hemodynamic \\ monitoring using transesophageal \\ echocardiography in orthopedic surgery
}

Background: Orthopedic surgery is commonly performed in elderly patients with higher cardiovascular surgical risk. Hemodynamic monitoring in these patients may prevent perioperative complications. Aim: To assess the usefulness of transesophageal echocargiography (TEE) for intraoperative cardiovascular monitoring during orthopedic surgery. Material and methods: Patients older than 65 years subjected to orthopedic surgery using general anesthesia and with a high cardiovascular risk were studied. Intraoperative TEE was performed to assess intravascular volume, myocardial contractility and the presence of myocardial ischemia. Results: Fifty three patients aged $72 \pm 5$ years were studied. Hemodynamic stability was detected in $68 \%$ of patients, mainly reflected as episodes of hypovolemia. Myocardial ischemia was detected in two patients and embolic episodes in six (11\%). Conclusions: Intraoperative TEE was useful for the hemodynamic evaluation of patients with high cardiovascular risk (Rev Méd Chile 2008; 136: 1127-33).

(Key words: Aged; Echocardiography, transesophageal; Hemodynamics)

Recibido el 16 de enero, 2008. Aceptado el 29 de mayo, 2008.

No existen conflictos de interés de los autores y esta investigación no contó con financiamiento externo.

${ }^{1}$ Sección Anestesiología, Hospital Clínico Fuerza Aérea de Chile. Santiago, Chile. ${ }^{2}$ Universidad de Valparaíso. Valparaíso, Chile.

$\mathrm{E}^{1}$ aumento de la edad y calidad de vida de la población ha significado que un mayor número de pacientes ancianos sean sometidos a cirugía y anestesia ${ }^{1,2}$. Una de las áreas que ha tenido un

Correspondencia a: Dra. María Carolina Cabrera Schulmeyer. Fernández Mira 796, Las Condes. Santiago, Chile. Teléfono: 56-2-7826164. Fax: 56-2-7826170. E mail: carol218@vtr.net gran desarrollo es la cirugía ortopédica, ya que el anciano y su médico traumatólogo al verlo comprometido en su quehacer físico, pero en óptimas condiciones mentales, buscan soluciones quirúrgicas a sus limitaciones y empeoramiento de calidad de vida. Una vez que se decide una cirugía de cadera, columna, hombro o rodilla, entre otras, es cuando se inicia un desafío real para el anestesiólogo. Esto se debe a que se trata en muchos casos 
de pacientes portadores de patologías crónicas como cardiopatía coronaria, hipertensiva, valvulopatías y diabetes. En condiciones de reposo y basales es muy probable evaluar y encontrar que el anciano está en una situación compensada, pero estas patologías alteran la reserva funcional de los diferentes parénquimas, lo que determina su fragilidad frente al estrés quirúrgico. El problema se plantea frente al uso de anestesia, la cirugía y las eventuales descompensaciones en su estado hemodinámico. ¿Cómo monitorizar a esta subpoblación de enfermos ancianos con riesgo cardiovascular aumentado, de tal manera de no alterar 0 empeorar su calidad de vida previa debido a un evento hemodinámico adverso durante el intraoperatorio?

Está bastante bien demostrado que patologías como la cardiopatía coronaria e hipertensiva dañan la distensibilidad del músculo miocárdico alterando la curva de correlación presión - volumen $^{3}$. Es así que en este tipo específico de pacientes las mediciones de presión (venosa central, arteria pulmonar y capilar pulmonar) no tienen una buena correlación con los valores de volumen. Se ha planteado serias dudas frente a la real utilidad de estas técnicas invasivas para monitorización cardiovascular intraoperatoria ${ }^{4,5}$.

Las causas del deterioro en la hemodinamia durante la cirugía ortopédica pueden deberse a diversas etiologías, como hipovolemia, el uso de fármacos, la presencia de isquemia miocárdica e incluso a fenómenos embólicos. El punto principal es el diagnóstico diferencial entre estas causas, ya que el enfrentamiento y terapia serán absolutamente diferentes dependiendo del diagnóstico. Es en este escenario donde aparece la ecocardiografía transesofágica intraoperatoria (ETE) como un interesante, novedoso y seguro sistema de monitorización hemodinámico ${ }^{6-9}$. La ETE es un monitor mínimamente invasivo, de rápida y fácil instalación, cuya principal ventaja es permitir observar en forma directa el corazón y sus grandes vasos y así, eventualmente, diagnosticar la etiología del compromiso hemodinámico. Con las imágenes ecocardiográficas es posible estimar los volúmenes y calcular en base a los gradientes que generan estos volúmenes en las cámaras, las presiones intracavitarias. Así en esta población de pacientes ancianos portadores de patología cardiaca que muy probablemente tienen alterada la distensibilidad ventricular, la ETE puede ser de muy alta utilidad en su monitorización intraoperatoria. La ETE permitiría obtener estimaciones de volúmenes, presiones y diagnósticos frente al compromiso agudo del estado hemodinámico mediante la visualización directa del corazón y sus grandes vasos. Así, el objetivo principal que se plantea en esta investigación es evaluar la real utilidad que pudiese aportar la ETE como monitor del estado hemodinámico durante cirugía ortopédica en pacientes ancianos con factores de riesgo cardiovascular aumentado.

\section{PACIENTES Y MÉTODOS}

El proyecto de monitorización hemodinámica con ETE fue presentado y aprobado por el Comité de Ética de Hospital. Todos los pacientes firmaron consentimiento informado.

En forma prospectiva entre enero de 2000 y diciembre de 2007 se estudiaron pacientes mayores de 65 años sometidos a cirugía ortopédica, con factores de riesgo cardiovascular aumentado. Los criterios de inclusión se basaron en las guías norteamericanas de ecocardiografía transesofágica intraoperatoria publicadas en $1996^{10}$. También se consideró para ingresar al estudio a aquellos pacientes que, según su evaluación preanestésica, fueron categorizados con riesgo cardiovascular aumentado por la presencia de una o más de las siguientes patologías: cardiopatía coronaria, cardiopatía hipertensiva, patología valvular aórtica 0 mitral moderada a severa, insuficiencia cardiaca con fracción de eyección baja $(<30 \%)$ e hipertensión pulmonar. Se excluyeron enfermos con patología gastroesofágica aguda, cirugía reciente esófago gástrica y presencia de várices esofágicas sangrantes.

Todos los pacientes fueron monitorizados con ETE luego de la inducción de anestesia general. Ésta se realizó utilizando remifentanilo, etomidato y un relajante neuromuscular no depolarizante. Luego de la intubación orotraqueal se introdujo el transductor para la realización de ETE. Ésta fue realizada por un anestesiólogo con entrenamiento avanzado. La máquina de ETE fue inicialmente un HP Sonos 2500 con un transductor transesofágico multiplanar de $5 \mathrm{Mhz}$ y luego en equipo Sonosite Micromaxx, también con un transductor multipla- 
nar de 5 Mhz. Los casos clínicos fueron grabados en video y reportados en la ficha clínica.

El examen ecocardiográfico basal se basó en las guías propuestas por la Sociedad Americana de Anestesiología Cardiovascular ${ }^{11}$, luego se mantuvo el transductor en la visión medio esofágica de cuatro cámaras o en el plano transgástrico a nivel del eje corto del ventrículo izquierdo. Para las mediciones hemodinámicas objetivas se realizaron diferentes estimaciones ecocardiográficas en cada paciente. Como indicador de la volemia se utilizó la imagen del tamaño del ventrículo derecho (VD) y la medición del volumen de fin de diástole (VFD) del ventrículo izquierdo (VI). Se monitorizó la presencia de isquemia miocárdica y para detectarla se evaluaron 16 segmentos miocárdicos en diferentes proyecciones. Utilizando una escala de 1 a 4 , se le confirió un puntaje a la contracción de cada segmento. Se consideró como puntaje 1 a la contracción normal, puntaje 2 a la hipokinesia, 3 como akinesia y al puntaje 4 como diskinesia. Para estudiar la función diastólica del VI se usó Doppler pulsado a nivel de la válvula mitral donde se obtuvieron dos ondas. La primera onda "E" correspondió al llene pasivo del VI y la onda siguiente fue la onda " $\mathrm{A}$ " que correspondió al llene activo producido por la contracción auricu$\operatorname{lar}^{12}$. Según los diferentes patrones de las ondas se determinó el grado de compromiso de la diástole. A este nivel también se evaluó la contractilidad miocárdica y realizando un trazado del volumen diastólico final (VDF) y del volumen sistólico final (VSF), se calculó su fracción de eyección (FE) con la siguiente fórmula:

$$
\mathrm{FE} \%=(\mathrm{VDF}-\mathrm{VSF} / \mathrm{VDF}) \times 100
$$

A nivel del tracto de entrada del ventrículo derecho (VD) se estimó la presión sistólica de la arteria pulmonar (PSAP). Para estimar la PSAP se evaluó la presencia de reflujo tricuspídeo con Doppler color y se midió su velocidad máxima con Doppler continuo. Con este valor y utilizando la fórmula simplificada de Bernoulli $\left(\mathrm{P}=4 \mathrm{x} \mathrm{v}^{2}\right)$ se estimó la PSAP.

En el plano transgástrico profundo, se midió la velocidad del tracto de salida del VI y así se calculó el gasto cardiaco aórtico. Para obtener este valor se realizó planimetría de la válvula aórtica y así se obtuvo su área. Luego se midió la velocidad máxima del tracto de salida del VI y se le calculó su integral (IVT). Al multiplicar el área valvular aórtica por la integral de la velocidad se obtuvo el volumen sistólico, que a su vez se multiplicó por la frecuencia cardiaca, obteniéndose el GC.

Luego de finalizado el estudio con ETE cada anestesiólogo evaluó su utilidad y la clasificó en una de los siguientes 4 grupos:

Grupo 1 = Las mediciones y estimaciones con ETE fueron principalmente útiles para el manejo de volúmenes, presiones y gasto cardiaco intraoperatorios.

Grupo 2 = La ETE fue útil en la indicación y en el manejo de fármacos intraoperatorios (ajuste de fármacos anestésicos y drogas vasoactivas).

Grupo 3 = Con las imágenes se realizó un nuevo diagnóstico que cambió el manejo anestésico.

Grupo 4 = La ETE se utilizó como sustituto del catéter de arteria pulmonar (según la evaluación preanestésica se habría considerado la instalación de catéter de Swan Ganz, pero ésta se desestimó luego de la utilización de ecografía durante el intraoperatorio. No siendo necesaria su instalación tampoco su instalación en el periodo postoperatorio).

Estadística. En la descripción estadística se calculó promedio, desviación estándar (DE) y porcentajes.

\section{RESULTADOS}

Se monitorizaron con ETE durante el intraoperatorio de cirugía ortopédica un total de 53 pacientes. La edad promedio fue de $72 \pm 5$ años (Tabla 1). Durante todo el periodo intraoperatorio, se monitorizaron 33 cirugías de cadera [10 artroplastias totales, 5 recambios de prótesis de cadera y quince clavos dinámicos endomedulares (DHS) ], 6 prótesis totales de rodilla, 9 cirugías de hombro y cinco cirugías de columna. Las patologías que determinaron la indicación de ETE intraoperatoria fueron en

\section{Tabla 1. Características de la población}

\begin{tabular}{|ll|}
\hline Número de pacientes & 53 \\
Edad (años) $\pm \mathrm{DE}$ & $72 \pm 5$ \\
Género & 20 mujeres y 33 varones \\
Peso $(\mathrm{kg})$ & $63,4 \pm 11$ \\
Talla $(\mathrm{m})$ & $1,61 \pm 10$ \\
\hline
\end{tabular}


el más alto porcentaje de los casos la cardiopatía coronaria o hipertensiva (Tabla 2). En todos los casos se obtuvieron imágenes adecuadas con ETE.

En 42 enfermos se registraron eventos hemodinámicos y en todos la ETE fue útil para el manejo. Según la evaluación del anestesiólogo encargado del paciente, con mayor frecuencia la ETE le fue útil para evaluar y estimar la volemia, fracción de eyección y contractilidad miocárdica en forma directa (Tabla 3).

La causa más frecuente de alteraciones en el estado cardiovascular fue la hipotensión. Esta se debió en $54 \%$ de los episodios a hipovolemia que fue corregida con éxito basándose en las imágenes y mediciones de área de fin de diástole del VI. A catorce pacientes se les realizó un nuevo diagnóstico que cambió su manejo anestésico (Tabla 4). Se visualizaron fenómenos embólicos en 6 enfermos. La presencia de embolización se observó en $100 \%$ de los casos que requirieron cementación de la prótesis de cadera (Figura 1). El grado de compromiso hemodinámico fue superior en los pacientes que presentaron fenómenos embólicos más severos. En un paciente se determinó que la causa de la hipotensión se debió a la presencia de un movimiento sistólico anterior del velo mitral (SAM por su traducción del inglés) que determinó obstrucción del tracto de salida del VI. También la terapia fue exitosa basándose en el aporte de volumen y betabloqueo.

En dos enfermos se diagnosticó isquemia miocárdica intraoperatoria basándose en la presencia de defectos de la contractilidad segmentaria persistentes durante todo el intraoperatorio.

No hubo complicaciones con el uso de ETE intraoperatorio en esta serie.

\section{DisCUSIÓN}

La ecocardiografía transesofágica intraoperatoria demostró ser un monitor eficiente y seguro para la evaluación hemodinámica de pacientes ancianos con riesgo cardiovascular aumentado sometidos a cirugía ortopédica. Sus principales beneficios fueron su bajo grado de invasividad, la excelente calidad de imágenes del corazón y sus grandes vasos y la baja incidencia de complicaciones. Sus grandes ventajas, al compararlo con otros métodos de monitorización cardiovascular, fueron que permitió evaluar en forma directa los volúmenes intracardiacos, diagnosticar la etiología del com-

\section{Tabla 2. Patologías cardiovasculares previas de los pacientes monitorizados con ecocardiografía transesofágica intraoperatoria}

\begin{tabular}{|lcc|}
\hline & $\mathrm{n}=53$ & $\%$ \\
\hline Cardiopatía coronaria & 29 & 54 \\
Cardiopatía hipertensiva & 21 & 39 \\
Fracción de eyección baja & 12 & 22 \\
Enfermedad mitral (insuficiencia o estenosis) & 12 & 22 \\
Enfermedad aórtica (insuficiencia o estenosis) & 11 & 20 \\
Hipertensión pulmonar & 3 & 6 \\
\hline
\end{tabular}

Tabla 3. Evaluación de utilidad de ecocardiografía transesofágica intraoperatoria según el anestesiólogo

\begin{tabular}{|llll|}
\hline & & $\mathrm{n}=53$ & $\%$ \\
\hline Grupo 1 & Estimaciones fueron útiles para manejo de volúmenes & 17 & 32 \\
Grupo 2 & Estimaciones fueron útiles para manejo de fármacos & 10 & 18 \\
Grupo 3 & Nuevos diagnósticos & 14 & 26 \\
Grupo 4 & Sustituto de catéter de arteria pulmonar & 12 & 22 \\
\hline
\end{tabular}




\section{Tabla $4 \mathrm{~N}$ uevos diagnósticos realizados con ecocardiografía transesofágica intraoperatoria (Grupo 3)}

\begin{tabular}{|ll|}
\hline & $\mathrm{n}$ \\
\hline Embolización pulmonar & 6 \\
Isquemia miocárdica & 2 \\
Estenosis mitral & 3 \\
Estenosis aórtica & 1 \\
Comunicación interauricular & 1 \\
Trombo en orejuela izquierda & 1 \\
\hline
\end{tabular}

promiso hemodinámico y con esto indicar terapias específicas. Si bien el mayor número de pacientes fue ubicado en el grupo 1 (esto es que la ETE fue especialmente útil en la evaluación de volúmenes, presiones y GC), en todos los casos prestó información de valor para el anestesiólogo. Estos resultados se comparan perfectamente con los que ya había obtenido nuestro grupo de trabajo monitorizando y manejando con ecocardiografía pacientes durante cirugía no cardiaca ${ }^{13}$.

La ETE permite estimar en forma directa los volúmenes intracardiacos y no inferirlos desde las mediciones de presión, que es la forma de obtenerlo desde el catéter de arteria pulmonar o desde el catéter venoso central. Esto cobra real importancia en los ventrículos con alteraciones de distensibilidad, como en la cardiopatía coronaria, hipertensiva y en los pacientes portadores de valvulopatías crónicas izquierdas. Es en estos casos donde la orientación y guía terapéutica estaría equivocada si se utilizan los datos de presión aportados por el catéter de arteria pulmonar o venoso central.

Con el uso de ecocardiografía en esta serie de pacientes se modificaron terapias y se guió el aporte de fluidos en forma exacta y acotada. Esta experiencia positiva coincide con otras publicaciones. Kolev y cols en un estudio multicéntrico europeo demostraron que el uso de ETE en 224 pacientes influenció las decisiones del manejo de terapia antiisquémica y de fluidos ${ }^{14}$.

Denault también demostró la utilidad de la ETE en una serie de 214 enfermos en que en base a los hallazgos intraoperatorios se realizaron nuevos diagnósticos ${ }^{15}$.

En la actualidad la ETE es el único monitor disponible para el anestesiólogo que permite visualizar y así confirmar fenómenos embólicos durante

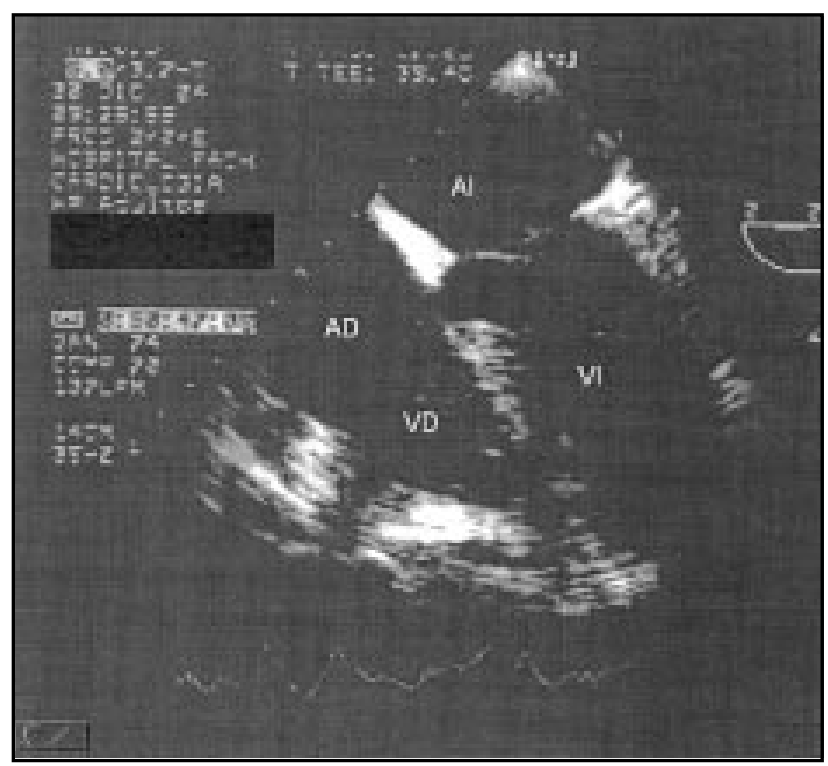

Figura 1. Imagen de ecocardiografía transesofágica intraoperatoria que muestra las cuatro cámaras cardiacas sin material ecogénico $(\mathrm{AD}=$ =aurícula derecha, $\mathrm{VD}$ =ventrículo derecho, $\mathrm{AI}$ =aurícula izquierda, $\mathrm{VI}$ =ventrículo izquierdo). 


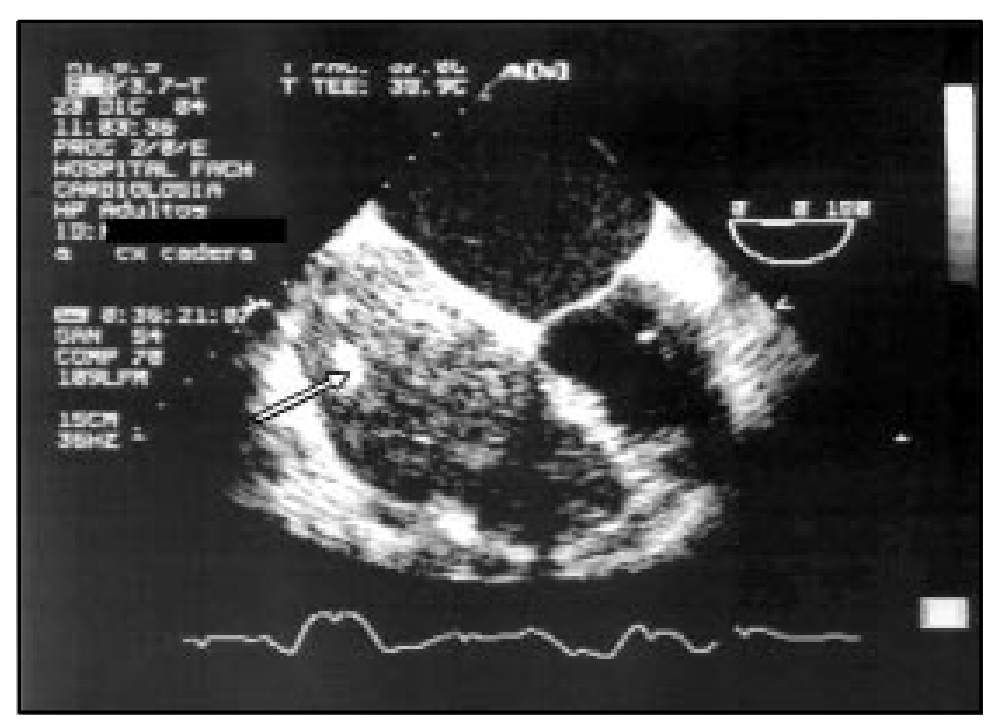

Figura 2. Imagen de cuatro cámaras cardiacas. La flecha muestra la presencia de abundante material hiperrefringente en aurícula y ventrículo derechos.

el intraoperatorio ${ }^{16-19}$. La presencia de embolizaciones durante cirugía ortopédica, especialmente de cadera, es un fenómeno que durante muchos años se sospechó como causa de morbimortalidad intraoperatoria ${ }^{20}$. Existen varios reportes de muerte intraoperatoria en donde el informe anátomo patológico demuestra la presencia de embolización. Puede ser tema de controversia que en esta serie de enfermos ancianos sólo se utilizara anestesia general, especialmente si se considera la cirugía de cadera. Se ha discutido ampliamente en la literatura con respecto al uso de anestesia regional o general en la cirugía ortopédica de cadera ${ }^{21}$, no existiendo una conclusión definitiva. En esta serie se planteó siempre el uso de anestesia general, no sólo por la utilización de la sonda transesofágica, sino que también por el manejo más acotado de la hemodinamia en pacientes con riesgo cardiovascular aumentado, evitando el bloqueo simpático producido por una anestesia regional central. En nuestro centro, luego de la introducción de la ecocardiografía como parte del sistema disponible de monitorización hemodinámica, se administra anestesia general, si el paciente cumple con los requisitos para utilizar ETE durante su intraoperatorio.

La presencia de un anestesiólogo a cargo del paciente y administrando la anestesia y de otro anestesiólogo diferente realizando la ETE también es un tema de cierta controversia ${ }^{22}$. No pareciera necesario que esta situación sea estrictamente la ideal que se produzca. En un alto porcentaje de esta serie (68\%), el anestesiólogo fue único. Con la aparición de equipos más modernos, de menor tamaño y menor complejidad para accionar los diferentes comandos es posible realizar las evaluaciones ecocardiográficas de manera simple y fácil.

La adecuada monitorización intraoperatoria necesariamente debe influir en la morbimortalidad perioperatoria ${ }^{23}$. Como se trata de pacientes críticos y complejos, por su edad, sus patologías y cuadro clínico, es difícil analizar por separado un sistema de monitorización, ya que una sola acción difícilmente explique cambios en la morbimortalidad, que es siempre el objetivo final buscado. También con este número de pacientes no es posible llegar a esta conclusión, pero es probable que al continuar investigando y evaluando los resultados se logre demostrar este punto, con el uso de ecografía intraoperatoria. En un interesante artículo de Swaminathan y $\operatorname{cols}^{24}$ demostraron que la presencia ecocardiográfica de alteraciones de contractilidad de la pared miocárdica durante una cirugía cardiaca se relaciona con una mayor incidencia de eventos cardiacos postoperatorios a largo plazo.

En conclusión, la ecocardiografía transesofágica intraoperatoria es un sistema de alta utilidad 
para una población específica de pacientes con riesgo cardiovascular aumentado. La real influencia del uso de este tipo de monitorización en la morbimortalidad perioperatoria debe ser evaluada en un mayor número de pacientes. Pero, sin duda,

\section{REFERENCIAS}

1. Praugh DS. Anesthesic pitfalls in the elderly patient. J Am Coll Surg 2005; 200: 784-94.

2. LoAn D, Hyde B, Zwischenberger J. Perioperative management of special populations: The Geriatric Patient. Surg Clin North Am 2005; 85: 1259-66.

3. Kitzman D, Daniel K. Diastolic Heart failure in the Elderly. Clin Geriatr Med 2007; 23: 83-106.

4. HeRER C. Invasive hemodynamic monitoring, the aftermath of the ESCAPE Trial. Cardiol Clin 2007; 25: 565-71.

5. Spodick D. Pulmonary Artery Catheter: A long and tortuous controversy. Am J Med 2006; 119: e17.

6. Suriani RJ, Neustein S, Shore-Lesserson L, Konstadt S. Intraoperative transesophageal echocardiography during noncardiac surgery. J Cardiothorac Vasc Anesth 1998; 12: 274-80.

7. Mishra M, Chauhan R, Sharma KK, Dhar A, Bhise M, DHOLE $S$ ET AL. Real-time intraoperative transesophageal echocardiography: how useful? Experience of 5,016 cases. J Cardiothorac Vasc Anesth 1998; 6: 625-32.

8. KALMEYER I. The safety of intraoperative transesophageal echocardiography: a case series of 7200 cardiac surgical patients. Anesth Analg 2001; 92: 1126-30.

9. Fenshane M, Euis C, Habib S, Konstadt S, Reich L A retrospective analysis of the costs and benefits related to alterations in cardiac surgery from routine intraoperative transesophageal echocardiography. Anesth Analg 2002; 95: 824-7.

10. Shanewise J, Cheung A, Aronson S, Steward W, Weiss R, MarK J ET AL. Practice Guidelines for perioperative transesophageal echocardiography. Anesthesiology 1996; 84: 986-1006.

11. Shanwise J, Cheung A, Aronson S, Steward W, Weiss $R$, MARK S ET AL. ASE/SCA guidelines for performing a comprehensive multiplane transesophageal echocardiography examination. Anesth Analg 1999; 89: 870-84.

12. Khouri S, Maly G, Suh D, Walsh T. A practical approach to the echocardiographic evaluation of diastolic function. Am J Cardiology 2004; 94: 123-34.

13. Cabrera MC, Santelices E, Schmied S, Vega R. Impact of intraoperative echocardiography in non cardiac surgery. J Cardiovasc Thor Surg 2006; 20: 768-71. la gran ayuda que significa visualizar y diagnosticar con ETE durante el intraoperatorio las etiologías que desencadenan diferentes cambios en el estado hemodinámico, son un gran avance en la seguridad de la práctica anestesiológica diaria.

14. Kolev N, Brase R, Swanevelder J. The influence of transoesophageal echocardiography on intra-operative decision making. A European multicentre study. European Perioperative TOE Research Group. Anaesthesia 1998; 53: 767-73.

15. Couture P, Denault AY, McKenty S, Boudrelaaut D, Plante F, Peron R et al. Impact of routine use of intraoperative transesophageal echocardiography during cardiac surgery. Can J Anesth 2000; 47: 20-6.

16. Murphy P, Edeust G, Byrick RJ, Kay JC, Mulen JB. Relationship of fat embolism to haemodynamic and echocardiographic changes during cemented arthroplasty. Can J Anaesth 1997; 44: 1293-300.

17. Orsini EC, Byrick RJ, MuLen BM. Cardiopulmonary function and pulmonary microemboli during arthroplasty using cemented or non-cemented components. J Bone Joint Surg Am 1987; 69: 822-32.

18. Koessler MJ, Fabiani $R$, Hamer $H$, Pitto RP. The clinical relevance of embolic events detected by transesophageal echocardiography during cemented hip arthroplasty: a randomized clinical trial. Anesth Analg 2001; 92: 49-55.

19. Lafont N, Kalonit M, Barre J, Guiliaume C, Boogaerts JC. Clinical features and echocardiography of embolism during cemented hip arthroplasty. Can J Anaesth 1997; 44: 112-7.

20. FaLON KM, FuLer JG, Moreiy-Forster P. Fat embolization and fatal cardiac arrest during hip arthroplasty with methylmethacrylate. Can J Anesth 2001; 48: 626-9.

21. Mauermann W, Shiley A, Zuo Z. A comparison of neuriaxial block versus general anesthesia for elective total hip replacement, a metaanalysis. Anesth Analg 2006; 103: 1018-25.

22. ARonson S, Thys DM. Training and certification in perioperative transesophageal echocardiography: a historical perspective. Anesth Analg 2001; 93: 1422-7.

23. Mayfield JB. The impact of intraoperative monitoring on patient safety. Anesthesiol Clin 2006; 24: 407-17.

24. Swaminathan M, Morris R, De Meyts D, Podgorenau M, JoLS J, Grocott H ET AL. Deterioration of regional wall motion immediately after coronary artery graft surgery is associated with long-term major adverse cardiac events. Anesthesiology 2007; 107: 739-45. 